\title{
INVESTIGATION OF SCALING PROPERTIES OF HYSTERESIS IN FINEMET THIN FILMS
}

\author{
L. Santi ${ }^{\text {a,c }}$, L. S. Dorneles ${ }^{\text {a }}$, R.L.Sommer ${ }^{\text {a }}$, F.Colaiori ${ }^{\text {b }}$, S. Zapperi ${ }^{\text {b }}$, A. Magni ${ }^{\text {, }}$, and \\ G. Durin ${ }^{\mathrm{c}, *}$ \\ ${ }^{a}$ Univ. Federal de Santa Maria, Dep. de Fisica, UFSM, 97105-900, Santa Maria, RS, Brasil \\ bINFM Unità di Roma 1, Dip. di Fisica, Università "La Sapienza", P.le A. Moro 2, 00185 Roma, Italy \\ ${ }^{\mathrm{c}}$ IEN Galileo Ferraris, str. delle Cacce 91, 10135 Torino, Italy
}

\begin{abstract}
We study the behavior of hysteresis loops in Finemet $\mathrm{Fe}_{73.5} \mathrm{Cu}_{1} \mathrm{Nb}_{3} \mathrm{Si}_{18.5} \mathrm{~B}_{4}$ thin films by using a fluxometric setup based on a couple of well compensated pickup coils. The presence of scaling laws of the hysteresis area is investigated as a function of the amplitude and frequency of the applied field, considering sample thickness from about $20 \mathrm{~nm}$ to $5 \mu \mathrm{m}$. We do not observed any scaling predicted by theoretical models, while dynamic loops show a logarithmic dependence on the frequency.
\end{abstract}

Key words: Dynamic hysteresis, thin films, magnetization processes PACS:

The hysteresis properties of magnetic thin and ultrathin films has been extensively investigated both theoretically and experimentally $[1,2,3]$. In view of their application in magnetic devices, it is fundamental to understand the microscopical origin of magnetization processes, especially considering the detailed features of domain wall motion and domain nucleation. Large part of present literature has analyzed the behavior of dynamic hysteresis measured by using the longitudinal magnetooptical Kerr effect (MOKE). Following many theoretical studies, data are analyzed in order to verify the existence of universal scaling laws. In particular, the dynamic hysteresis loop area $A$ is assumed

* Corresponding author. Tel.: +39 0113919841; fax: +39 0113919834; e-mail: durin@ien.it to follow a law of the type $A \sim A_{0}+H_{0}^{\alpha} \omega^{\beta}$, where $A_{0}$ is the static hysteresis, $H_{0}$ and $\omega$ are the amplitude and the frequency of the applied field, and $\alpha, \beta$ two critical exponents. As a matter of fact, the estimated critical exponents span a quite large range, so that the true existence of scaling laws and universality is still under question. Beside this, a direct connection between experimental observations and the underlying magnetization processes cannot be still provided.

We investigate the dynamical hysteresis properties from a technique complementary to the MOKE. Using a couple of well compensated sets of coils, we detect the induced flux rate, which is proportional to the volume magnetization change. On the contrary, the MOKE technique detects 


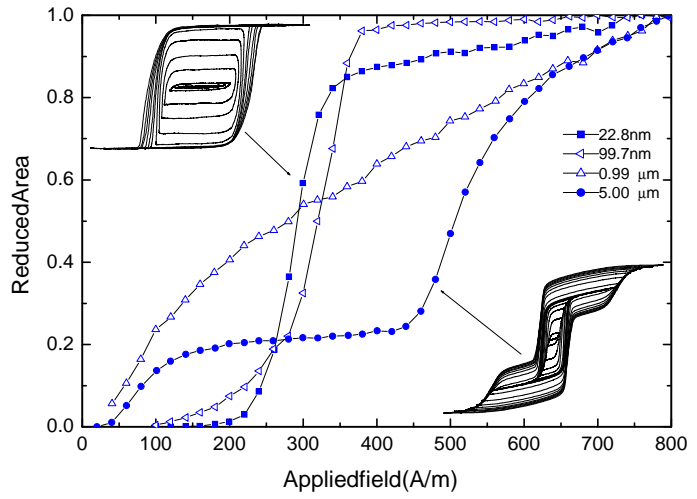

Fig. 1. Hysteresis loop area for Finemet thin films at different thicknesses. The area is divided by the value at $\mathrm{H}=800$ $\mathrm{A} / \mathrm{m}$ for convenience. Examples of minor loop measurements are shown for the thinnest $(22.8 \mathrm{~nm})$ and the thickest $(5 \mu \mathrm{m})$ sample.

the magnetization only on the sample surface and within the laser spot area. We believe that the use of both techniques helps to better investigate the magnetization processes, especially when the sample thickness can play an important role.

We consider a set of amorphous Finemet thin films of composition $\mathrm{Fe}_{73.5} \mathrm{Cu}_{1} \mathrm{Nb}_{3} \mathrm{Si}_{18.5} \mathrm{~B}_{4}$, with thickness ranging from about $20 \mathrm{~nm}$ to $5 \mu \mathrm{m}$, calibrated with low angle x-ray diffractometry. Lateral dimensions are about $1.5 \mathrm{~cm}$ by $3 \mathrm{~cm}$. The dependence of the hysteresis loop on the applied field amplitude is reported in Fig. 1 at four sample thickness. The data show very different behaviors, and simple power laws does not hold. At lower thickness, the loops are roughly squared, as shown in the upper left corner of Fig. 1, and the loop area steeply changes for applied field approaching the coercive field. Only at intermediate thickness, we can fit the data with a power law, getting $\alpha \sim$ 0.4. Finally, at larger thickness, the loops display a "double switching" structure (lower right corner), similar to whose reported for some spin-valve structures [4]: this behavior suggests the presence of two magnetic sub-systems having different coercive fields. We do not know the origin of this hysteresis, but we can confirm this is a common feature of Finemet samples having the same thickness, as we found similar loops for samples of slightly different composition.

A similar dependence on the sample thickness

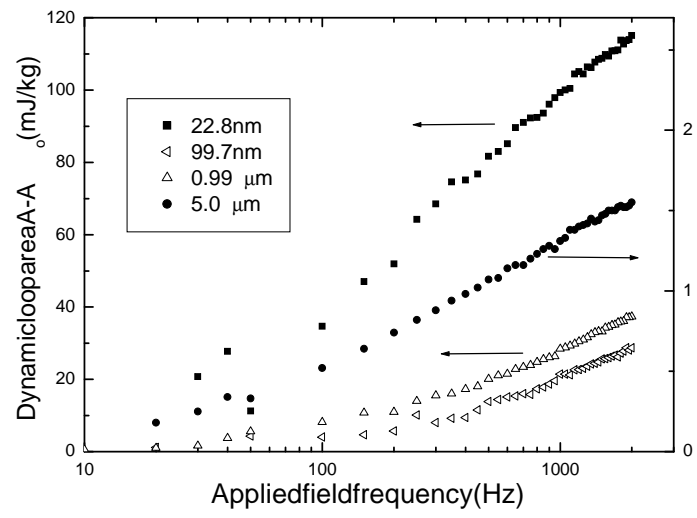

Fig. 2. Frequency dependence of the dynamic hysteresis area $A-A_{0}$ obtained by fluxometric measurements. Data for the thickest sample are obtained considering only the inner loops not showing the double switching as in Fig. 1.

is not found when considering the behavior of dynamic loops: in this case, all the hysteresis area roughly follow the logarithm of frequency, as shown in the semi-log plot of Fig. 2. It is interesting to observe the results for the $5 \mu \mathrm{m}$; the double switching loops are not much affected by the frequency, as we observe only a slight change in the curves around the coercive field. This is in contrast with the results in the spin-valves of Ref. [4] where the loops transform to a "single switch" behavior. We thus considered only the inner loops not showing the double switching, and found they also show the logarithmic dependence as the other samples, as shown in Fig. 2. Even if the data at low frequencies are rather noisy, as usual with this fluxometric technique, we cannot observe any clear dynamic transition in the hysteresis, which would imply a steep change of exponent $\beta$. All these results suggest that a more general approach, not only based on the existence of scaling laws, is needed to investigate the hysteresis properties in thin films.

\section{References}

[1] B. K. Chakrabarti and M. Acharyya, Rev. Mod. Phys. 71 (1999) 847.

[2] I. Ruiz-Feal et al., Phys. Rev. B 65(2002) 054409.

[3] H. Jang and M. J. Grimson, Phys. Rev. E 63 (2001) 066119. 
[4] W. Y. Lee et al., Phys. Rev. B 61 (2000) 6811. 